\title{
On a delay population model with quadratic nonlinearity
}

Leonid Berezansky ${ }^{1}$, Jaromír Baštinec ${ }^{2}$, Josef Diblík ${ }^{2,3 *}$ and Zdeněk Šmarda²

*Correspondence:

diblik.j@fce.vutbr.cz

${ }^{3}$ Department of Mathematics and

Descriptive Geometry, Faculty of

Civil Engineering and Department

of Mathematics, Faculty of Electrical

Engineering and Communication,

Brno University of Technology, Brno,

Czech Republic

Full list of author information is

available at the end of the article

\begin{abstract}
A nonlinear delay differential equation with quadratic nonlinearity,

$$
\dot{x}(t)=r(t)\left[\sum_{k=1}^{m} \alpha_{k} x\left(h_{k}(t)\right)-\beta x^{2}(t)\right], \quad t \geq 0,
$$

is considered, where $\alpha_{k}$ and $\beta$ are positive constants, $h_{k}:[0, \infty) \rightarrow \mathbb{R}$ are continuous functions such that $t-\tau \leq h_{k}(t) \leq t, \tau=$ const, $\tau>0$, for any $t>0$ the inequality $h_{k}(t)<t$ holds for at least one $k$, and $r:[0, \infty) \rightarrow(0, \infty)$ is a continuous function satisfying the inequality $r(t) \geq r_{0}=$ const for an $r_{0}>0$. It is proved that the positive equilibrium is globally asymptotically stable without any further limitations on the parameters of this equation.
\end{abstract}

\section{Introduction}

To include oscillation in population model systems, Hutchinson $[1,1948]$ suggested the following delay logistic equation:

$$
\frac{d N(t)}{d t}=r N(t)\left[1-\frac{N(t-\tau)}{K}\right]
$$

where $N(t)$ is the population size at time $t, r>0$ is the intrinsic growth rate of the population, $\tau>0$ and $K>0$ is the carrying capacity of the population.

There are many generalizations and modifications of Hutchinson's equation [2-7]. In particular, a delay logistic equation with several delays,

$$
\dot{x}(t)=x(t)\left[\alpha-\sum_{k=1}^{m} \beta_{k} x\left(t-\tau_{k}\right)\right],
$$

where $\alpha, \beta_{k}$ and $\tau_{k}>0$ are positive constants, is considered in [8, p.87]. Equation (1) can be viewed as one with quadratic nonlinearities of the unknown function $x$. For more work on the stability and boundedness of equations and systems related to (1), one can refer to [9-13].

(C) 2012 Berezansky et al.; licensee Springer. This is an Open Access article distributed under the terms of the Creative Commons Attribution License (http://creativecommons.org/licenses/by/2.0), which permits unrestricted use, distribution, and reproduction in any medium, provided the original work is properly cited. 
In the monograph [14, p.177], the author considers the following population model with quadratic nonlinearity:

$$
\dot{x}(t)=\sum_{k=1}^{m} \alpha_{k} x\left(t-\tau_{k}\right)-\beta x^{2}(t), \quad t \geq 0,
$$

where $\alpha_{k}>0, \beta>0, \tau_{k}>0$, and with the initial condition

$$
x(t)=\varphi(t), \quad t \in\left[-\tau^{*}, 0\right]
$$

where $\varphi:\left[-\tau^{*}, 0\right] \rightarrow \mathbb{R}$ is a continuous function, $\tau^{*}=\max _{k=1, \ldots, m} \tau_{k}$ and $\varphi(t)>0$ if $t \in$ $\left[-\tau^{*}, 0\right]$.

As can simply be verified, equation (2) has a unique positive equilibrium $x(t)=K, t \in$ $\left[-\tau^{*}, \infty\right)$, where

$$
K=\frac{\alpha}{\beta}, \quad \alpha:=\sum_{k=1}^{m} \alpha_{k} .
$$

Theorem 1 [14, Corollary 3.2.2, p.177] The positive equilibrium $K$ is a global attractor of problem (2), (3).

This result is different from almost all known results on the stability for nonlinear delay differential equations since there are no limitations on the parameters of equation (2). The proof of Theorem 1 is based on the method of Lyapunov-Krasovskii functionals.

Consider the nonautonomous equation with quadratic nonlinearity

$$
\dot{x}(t)=r(t)\left[\sum_{k=1}^{m} \alpha_{k} x\left(h_{k}(t)\right)-\beta x^{2}(t)\right], \quad t \geq 0,
$$

where $\alpha_{k}>0, \beta>0, h_{k}:[0, \infty) \rightarrow \mathbb{R}$ are continuous functions such that the inequalities $t-\tau \leq h_{k}(t) \leq t$ are true for a $\tau=$ const, $\tau>0$, and $r:[0, \infty) \rightarrow(0, \infty)$ is a continuous function satisfying the inequality $r(t) \geq r_{0}=$ const for an $r_{0}>0$. We suppose also that for any $t>0$ the inequality $h_{k}(t)<t$ holds for at least one $k$.

Together with (5), we consider an initial problem

$$
x(t)=\varphi(t), \quad t \in[-\tau, 0],
$$

where $\varphi:[-\tau, 0] \rightarrow \mathbb{R}$ is a continuous function and $\varphi(t)>0$ if $t \in[-\tau, 0]$.

It is obvious that equation (2) is a particular case of equation (5) if we set

$$
r(t):=1, \quad h_{k}(t):=t-\tau_{k}, \quad \tau:=\tau^{*} .
$$

Moreover, it is easy to see that the constant $K$ defined by (4) defines the unique positive equilibrium $x(t)=K, t \in[-\tau, \infty)$ of equation (5) as well.

In this paper, we prove that there exists a unique positive global solution to the problem (5), (6). Let us recall that a function $x:[-\tau, \infty) \rightarrow \mathbb{R}$ continuous on $[-\tau, \infty)$ and continuously differentiable on $[0, \infty)$ is called a global solution to the problem (5), (6) if it satisfies 
equation (5) on $[0, \infty)$ and initial condition (6). In addition to this, we prove that this solution is a bounded function isolated from zero. Finally, we will extend Theorem 1 to nonautonomous equation (5). Unfortunately, the construction of a Lyapunov-Krasovskii functional for autonomous equation (2) given in [14] to prove Theorem 1 is not applicable to nonautonomous equation (5). Therefore, we use another method based on a special quasi-linearization of a given nonlinear equation.

We will apply the following standard notions given in the definition below.

Definition 1 The equilibrium solution $x(t)=K, t \in[-\tau, \infty)$ of equation (5) is locally stable if, for any $\varepsilon>0$, there exists $\delta>0$ such that the inequality $|\varphi(t)-K|<\delta, t \in[-\tau, 0]$ implies $|x(t)-K|<\varepsilon, t \geq 0$ if $x(t)=\varphi(t), t \in[-\tau, 0]$. If, in addition, for any such solution, $\lim _{t \rightarrow \infty} x(t)=K$, the equilibrium solution $x(t)=K, t \in[-\tau, \infty)$ of equation (5) is called locally asymptotically stable.

The equilibrium solution $x(t)=K, t \in[-\tau, \infty)$ of equation (5) is called a global attractor of equation (5) if $\lim _{t \rightarrow \infty} x(t)=K$ for all solutions $x(t)$ of equation (5) defined by all initial functions described by (6).

The equilibrium solution $x(t)=K, t \in[-\tau, \infty)$ of equation (5) is globally asymptotically stable if it is a global attractor for all solutions $x(t)$ of equation (5) defined by all initial functions described by (6) and if it is also locally stable.

\section{Main results}

In this section, we employ a simple stability result to the following linear equation:

$$
\dot{x}(t)+a(t) x(t)+\sum_{k=1}^{m} b_{k}(t) x\left(h_{k}(t)\right)=0, \quad t \geq 0,
$$

with bounded continuous functions

$$
a:\left[t_{0}, \infty\right) \rightarrow \mathbb{R}, \quad b_{k}:\left[t_{0}, \infty\right) \rightarrow \mathbb{R}, \quad h_{k}:\left[t_{0}, \infty\right) \rightarrow \mathbb{R},
$$

where $t-\tau \leq h_{k}(t) \leq t, \tau>0$, and for any $t>0$, the inequality $h_{k}(t)<t$ holds for at least one $k$.

Lemma 1 Assume that there exists a constant $a_{0}>0$ such that

$$
a(t) \geq a_{0}, \quad t \in[0, \infty)
$$

and

$$
\limsup _{t \rightarrow \infty} \frac{\sum_{k=1}^{m}\left|b_{k}(t)\right|}{a(t)}<1
$$

Then equation (7) is asymptotically stable.

This result has a long history. The first stability conditions of this kind were obtained by Krasovskii [15] for an equation with a single delay. A weaker result (a corollary of Lemma 1) is given in [16, p.154]. Lemma 1 itself is a consequence of [17, Corollary 3.13]. 
Theorem 2 A solution of problem (5), (6) is positive and global.

Proof Since $\varphi(0)>0$, there exists a unique positive local solution of problem (5), (6). Suppose [0,c) with $c>0$ is the maximum interval of existence of this problem and a point $t_{0} \in(0, c)$ is such that $x(t)>0, t \in\left[-\tau, t_{0}\right)$, and $x\left(t_{0}\right)=0$. Then $\dot{x}\left(t_{0}\right) \leq 0$. Directly from equation (5), we get

$$
\dot{x}\left(t_{0}\right)=r\left(t_{0}\right) \sum_{k=1}^{m} \alpha_{k} x\left(h_{k}\left(t_{0}\right)\right)>0,
$$

which is a contradiction.

Hence, $x(t)>0, t \in[0, c)$. If $c=\infty$, the theorem is proved. Suppose $c<\infty$. Then, by [16, Corollary 3.1, p.45], either $\lim \sup _{t \rightarrow c_{-}} x(t)=+\infty$ or $\liminf _{t \rightarrow c-} x(t)=0$.

Let $\lim \sup _{t \rightarrow c_{-}} x(t)=+\infty$. Then we have

$$
\dot{x}(t) \leq r(t) \sum_{k=1}^{m} \alpha_{k} x\left(h_{k}(t)\right), \quad t \in[0, c) .
$$

Corollary 2.4 [18, p.32] implies that $x(t) \leq y(t)$, where $y$ is a solution of the initial linear problem

$$
\begin{aligned}
& \dot{y}(t)=r(t) \sum_{k=1}^{m} \alpha_{k} y\left(h_{k}(t)\right), \quad t \in[0, \infty), \\
& y(t)=x(t), \quad t \in[-\tau, 0] .
\end{aligned}
$$

Since every solution $y$ of a linear delay differential equation is bounded on any finite interval, $x$ is also bounded on the interval $[0, c)$. We have a contradiction.

Suppose now $\liminf _{t \rightarrow c-} x(t)=0$. Then either $x$ is a monotone decreasing function on $\left[t_{0}, c\right)$ for some $t_{0} \in[0, c)$ or there exists a sequence $t_{n} \in\left[t_{0}, c\right)$ such that

$$
t_{n}<t_{n+1}, \quad \lim _{n \rightarrow \infty} t_{n}=c, \quad \lim _{n \rightarrow \infty} x\left(t_{n}\right)=0, \quad \dot{x}\left(t_{n}\right)=0
$$

and

$$
x(t)>x\left(t_{n}\right), \quad t \in\left[-\tau, t_{n}\right) .
$$

In the first case (without loss of generality, we assume that $x$ is a monotone decreasing function for $t \in[0, c)), \lim _{t \rightarrow c} x(t)=0$. Hence, $x$ is a solution of (5), (6) on $[0, c]$ with $x(c)=0$ and as above, we obtain a contradiction because

$$
0 \geq \dot{x}(c)=r(c) \sum_{k=1}^{m} \alpha_{k} x\left(h_{k}(c)\right)>0 .
$$

Consider now the second case. Then

$$
0=\dot{x}\left(t_{n}\right)=r\left(t_{n}\right)\left[\sum_{k=1}^{m} \alpha_{k} x\left(h_{k}\left(t_{n}\right)\right)-\beta x^{2}\left(t_{n}\right)\right] .
$$


Let $n \rightarrow \infty$. Then $t_{n} \rightarrow c$, hence

$$
\sum_{k=1}^{m} \alpha_{k} x\left(h_{k}(c)\right)=0 \text {, }
$$

where for at least one $k$ one has $h_{k}(c)<c$, and so $x\left(h_{k}(c)\right)=0$. We have a contradiction. The theorem is proved.

Theorem 3 For a solution $x$ of problem (5), (6),

$$
\limsup _{t \rightarrow \infty} x(t)<\infty, \quad \liminf _{t \rightarrow \infty} x(t)>0
$$

Proof Substituting $x(t)=y(t)+K$, equation (5) takes the form

$$
\dot{y}(t)=r(t)\left[\sum_{k=1}^{m} \alpha_{k} y\left(h_{k}(t)\right)-2 \alpha y(t)-\beta y^{2}(t)\right] .
$$

Hence,

$$
\dot{y}(t) \leq r(t)\left[\sum_{k=1}^{m} \alpha_{k} y\left(h_{k}(t)\right)-2 \alpha y(t)\right] .
$$

Consider the linear equation

$$
\dot{z}(t)=r(t)\left[\sum_{k=1}^{m} \alpha_{k} z\left(h_{k}(t)\right)-2 \alpha z(t)\right] \text {, }
$$

where

$$
z(t)=y(t), \quad t \in[-\tau, 0] .
$$

Corollary 2.4 [18, p.32] implies that $y(t) \leq z(t)$ for $t>0$. Now, we apply Lemma 1 to equation (9). Since

$$
\frac{r(t) \sum_{k=1}^{m} \alpha_{k}}{2 r(t) \alpha}=\frac{r(t) \alpha}{2 r(t) \alpha}=\frac{1}{2}<1,
$$

by Lemma 1, equation (9) is asymptotically stable. Hence, the function $y$ is bounded from above and consequently is the function $x$.

Suppose $\liminf _{t \rightarrow \infty} x(t)=0$. Then either $x$ is an eventually monotone decreasing function or there exists a sequence $t_{n} \in[-\tau, \infty)$ such that

$$
t_{n}<t_{n+1}, \quad \lim _{n \rightarrow \infty} t_{n}=\infty, \quad \dot{x}\left(t_{n}\right)=0
$$

and

$$
x(t)>x\left(t_{n}\right), \quad t \in\left[-\tau, t_{n}\right) .
$$


In the first case (without loss of generality, we assume that $x$ is a monotone decreasing function for $t>0)$, there exists $t *>0$ such that $x\left(t_{*}\right)<K$. Hence,

$$
\begin{aligned}
\dot{x}\left(t_{*}\right) & =r\left(t_{*}\right)\left[\sum_{k=1}^{m} \alpha_{k} x\left(h_{k}\left(t_{*}\right)\right)-\beta x^{2}\left(t_{*}\right)\right] \\
& \geq r\left(t_{*}\right)\left[\sum_{k=1}^{m} \alpha_{k} x\left(t_{*}\right)-\beta x^{2}\left(t_{*}\right)\right]=r\left(t_{*}\right)\left[\alpha x\left(t_{*}\right)-\beta x^{2}\left(t_{*}\right)\right]>0 .
\end{aligned}
$$

This is in contradiction to our assumption.

Consider now the second case. Then there exists a sufficiently large integer $n$ such that $x\left(t_{n}\right)<K$. Hence,

$$
0=\dot{x}\left(t_{n}\right)=r\left(t_{n}\right)\left[\sum_{k=1}^{m} \alpha_{k} x\left(h_{k}\left(t_{n}\right)\right)-\beta x^{2}\left(t_{n}\right)\right]>r\left(t_{n}\right)\left[\alpha x\left(t_{n}\right)-\beta x^{2}\left(t_{n}\right)\right]>0 .
$$

We have a contradiction and the theorem is proved.

In the following, when discussing the stability properties of solutions of equation (5), we will assume that initial conditions (6) hold.

Theorem 4 The positive equilibrium $K$ of equation (5) is globally asymptotically stable.

Proof We have to prove that $K$ is an attractor for all solutions of the equation and that it also is locally stable. To do this, it is sufficient to prove that the zero solution is an attractor for all solutions of equation (8) and that it also is locally stable.

Suppose that $x$ is a fixed solution of equation (5). Then $y(t)=x(t)-K$ is a fixed solution of equation (8). After substituting $y(t)=e^{-\lambda t} z(t)$, where $\lambda>0$ is a sufficiently small number satisfying $\lambda<r_{0}(\alpha+\beta m)$, we have an equation

$$
\dot{z}(t)=r(t)\left[\sum_{k=1}^{m} \alpha_{k} e^{\lambda\left(t-h_{k}(t)\right)} z\left(h_{k}(t)\right)-\left(2 \alpha-\frac{\lambda}{r(t)}\right) z(t)-\beta e^{-\lambda t} z^{2}(t)\right] .
$$

Since $y(t)=e^{-\lambda t} z(t)$, we can rewrite equation (10) as

$$
\dot{z}(t)=r(t)\left[\sum_{k=1}^{m} \alpha_{k} e^{\lambda\left(t-h_{k}(t)\right)} z\left(h_{k}(t)\right)-\left(2 \alpha-\frac{\lambda}{r(t)}+\beta y(t)\right) z(t)\right] .
$$

By Theorem 3, there exist constants $m, M, 0<m<M$ such that

$$
m<x(t)<M
$$

for $t>0$ and

$$
m-K<y(t)<M-K \text {. }
$$

For $t>0$, we have

$$
2 \alpha-\frac{\lambda}{r(t)}+\beta y(t)>2 \alpha-\frac{\lambda}{r(t)}+\beta(m-K)=\alpha-\frac{\lambda}{r(t)}+\beta m
$$


and

$$
\sum_{k=1}^{m} \alpha_{k} e^{\lambda\left(t-h_{k}(t)\right)}<\alpha e^{\lambda \tau}
$$

Hence,

$$
\frac{\sum_{k=1}^{m} \alpha_{k} e^{\lambda\left(t-h_{k}(t)\right)}}{2 \alpha-\frac{\lambda}{r(t)}+\beta y(t)}<\frac{\alpha e^{\lambda \tau}}{\alpha-\frac{\lambda}{r(t)}+\beta m} \leq \frac{\alpha e^{\lambda \tau}}{\alpha-\frac{\lambda}{r_{0}}+\beta m}, \quad t \in[0, \infty) .
$$

Since

$$
\lim _{\lambda \rightarrow 0} \frac{\alpha e^{\lambda \tau}}{\alpha-\frac{\lambda}{r_{0}}+\beta m}=\frac{\alpha}{\alpha+\beta m}<1,
$$

for a sufficiently small $\lambda>0$,

$$
\limsup _{t \rightarrow \infty} \frac{\sum_{k=1}^{m} \alpha_{k} e^{\lambda\left(t-h_{k}(t)\right)}}{2 \alpha-\frac{\lambda}{r(t)}+\beta y(t)}<1
$$

We will now fix such $\lambda$. Suppose $u$ is an arbitrary continuous function such that $m-K<$ $u(t)<M-K$. By Lemma 1 , all solutions of linear equation (11), where $y$ is replaced by $u$, tend to zero. Then it is also true if $u=y$. Hence, $z$ as a solution of nonlinear equation (11) is a bounded function. Then

$$
\limsup _{t \rightarrow \infty} y(t)=\limsup _{t \rightarrow \infty} e^{-\lambda t} z(t)=0 \text {. }
$$

It means that the zero solution is a global attractor of equation (8). We need only to prove that this equation is locally stable. The linearized equation for (8) has the form

$$
\dot{u}(t)=r(t)\left[\sum_{k=1}^{m} \alpha_{k} u\left(h_{k}(t)\right)-2 \alpha u(t)\right] \text {. }
$$

By Lemma 1, equation (13) is asymptotically stable (see equation (9) in the proof of Theorem 3$)$. The theorem is proved.

Remark 1 The proof of Theorem 4 provides not only global asymptotic stability of the positive equilibrium $K$, but also exponential estimation of the rate of convergence of solutions. Tracing the proof carefully, we have

$$
x(t)-K=y(t)=e^{-\lambda t} z(t),
$$

where $x$ is a fixed solution of equation (5), $y(t)$ is a corresponding solution of equation (8) and $z(t)$ is a corresponding solution of equation (11). Since $z(t)$ is bounded and (12) holds, we state that for a given solution $x=x(t)$ of equation (5), there exists a constant $H_{x}$ such that

$$
|x(t)-K| \leq H_{x} e^{-\lambda t}
$$

where $\lambda$ is a sufficiently small positive number satisfying $\lambda<r_{0}(\alpha+\beta m)$ and $t \in[-\tau, \infty)$. 


\section{Conclusions, concluding remarks and open problems}

It is well known that the positive equilibrium $K$ of the delay logistic equation

$$
\dot{x}(t)=a x(t)\left(1-\frac{x(t-\tau)}{K}\right)
$$

is globally asymptotically stable if $a \tau<3 / 2$ and locally asymptotically stable if $a \tau<\pi / 2$. Thus, there is a gap between sufficient conditions for global and for local stabilities. One of the old problems is to show that local asymptotic stability implies global asymptotic stability for this equation. This problem also remains open for most of known nonlinear delayed equations in mathematical biology.

By Theorem 4, local and global stability conditions for equation (8) coincide since this equation is stable for all positive coefficients. It would be interesting to find other equations with such a property.

In the proof of Theorem 4, we applied the substitution $y(t)=z(t) e^{-\lambda t}$ with a small parameter $\lambda>0$. Such kind of substitutions are well known in the investigation of stability of linear equations. Maybe such a substitution is used here for a nonlinear equation for the first time.

The usefulness of asymptotic methods could be exploited to extend other powerful (deterministic) techniques such as the Laplace decomposition method or He's polynomials to deal with difference-differential models (see references $[19,20]$ for instance).

A partial case of equation (8) was considered in [21] where also some other delay differential equations with quadratic nonlinearity were studied.

Finally, we outline some open problems and topics for further research.

1. Study the oscillation properties of equation (5).

2. Extend Theorems 2-4 to the equation

$$
\dot{x}(t)=\sum_{k=1}^{m} a_{k}(t) x\left(h_{k}(t)\right)-b(t) x^{2}(t)
$$

if $\sum_{k=1}^{m} a_{k}(t)=b(t)$.

3. Prove the existence of a periodic solution for equation (14) with periodic functions $a_{k}(t), b(t)$ and study its local and global stability.

4. Study the existence, uniqueness, oscillation and stability properties of solutions of the following equations:

$$
\dot{x}(t)=\sum_{k=1}^{m} a_{k}(t) x\left(h_{k}(t)\right)-b(t) x^{n}(t), \quad n>0,
$$

and

$$
\dot{x}(t)=\sum_{k=1}^{m} a_{k}(t) x\left(h_{k}(t)\right)-b(t) x^{2}(g(t))
$$


Authors' contributions

The authors have made the same contribution. All authors read and approved the final manuscript.

\section{Author details}

'Department of Mathematics, Ben-Gurion University of the Negev, Beer-Sheva, 84105, Israel. ²Department of Mathematics, Faculty of Electrical Engineering and Communication, Brno University of Technology, Brno, Czech Republic. ${ }^{3}$ Department of Mathematics and Descriptive Geometry, Faculty of Civil Engineering and Department of Mathematics, Faculty of Electrical Engineering and Communication, Brno University of Technology, Brno, Czech Republic.

\section{Acknowledgements}

The second author was supported by Grant P201/10/1032 of the Czech Grant Agency (Prague). The third author was supported by the Operational Programme Research and Development for Innovations, No. CZ.1.05/2.1.00/03.0097, as an activity of the regional Centre AdMaS. The fourth author was supported by the Grant FEKT-S-11-2-921 of the Faculty of Electrical Engineering and Communication, Brno University of Technology. The authors would like to thank the referees for helpful suggestions incorporated into this paper

Received: 16 November 2012 Accepted: 3 December 2012 Published: 28 December 2012

\section{References}

1. Hutchinson, GE: Circular causal systems in ecology. Ann. N.Y. Acad. Sci. 50, 221-240 (1948-1950)

2. Faria, T: Asymptotic stability for delayed logistic type equations. Math. Comput. Model. 43(3-4), 433-445 (2006)

3. Faria, T, Liz, E: Boundedness and asymptotic stability for delayed equations of logistic type. Proc. R. Soc. Edinb., Sect. A, Math. 133(5), 1057-1073 (2003)

4. Lisena, B: Global attractivity in nonautonomous logistic equations with delay. Nonlinear Anal., Real World Appl. 9(1), 53-63 (2008)

5. Muroya, Y: Permanence, contractivity and global stability in logistic equations with general delays. J. Math. Anal. Appl. 302(2), 389-401 (2005)

6. Tang, XH: Global attractivity for a delay logistic equation with instantaneous terms. Nonlinear Anal. 59(1-2), 211-233 (2004)

7. Trofimchuk, E, Trofimchuk, S: Global stability in a regulated logistic growth model. Discrete Contin. Dyn. Syst., Ser. B 5(2), 461-468 (2005)

8. Györi, I, Ladas, G: Oscillation Theory of Delay Differential Equations. Oxford Science Publications (1991)

9. Khusainov, D, Agarwal, RP, Davidov, V: Stability and estimates for the convergence of solutions for systems involving quadratic terms with constant deviating arguments. Comput. Math. Appl. 38(11-12), 141-149 (1999)

10. Khusainov, D, Ivanov, A, Grytsay, I: Stability of delay systems with quadratic nonlinearities. Nonlinear Dyn. Syst. Theory 6(2), 159-169 (2006)

11. Kolmanovskii, $\mathrm{V}$, Myshkis, A: Introduction to the Theory and Applications of Functional-Differential Equations. Mathematics and Its Applications, vol. 463. Kluwer Academic, Dordrecht (1999)

12. Stević, S: Globally bounded solutions of a system of nonlinear functional differential equations with iterated deviating argument. Appl. Math. Comput. 219, 2180-2185 (2012)

13. Stević, S: Unique existence of bounded continuous solutions on the real line of a class of nonlinear functional equations with complicated deviations. Appl. Math. Comput. 218, 7813-7817 (2012)

14. Gopalsamy, K: Stability and Oscillations in Delay Differential Equations of Population Dynamics. Mathematics and Its Applications, vol. 74. Kluwer Academic, Dordrecht (1992)

15. Krasovskii, NN: Stability of Motion, Applications of Lyapunov's Second Method to Differential Systems and Equations with Delay. Translated by J.L. Brenner. Stanford University Press, Stanford (1963)

16. Hale, J, Verduyn, LS: Introduction to Functional Differential Equations. Springer, New York (1993)

17. Berezansky, L, Braverman, E: New stability conditions for linear differential equations with several delays. Abstr. Appl. Anal. 2011, Article ID 178568 (2011). doi:10.1155/2011/178568

18. Agarwal, RP, Berezansky, L, Braverman, E, Domoshnitsky, A: Nonoscillation Theory of Functional Differential Equations with Applications. Springer, New York (2012)

19. Hussain, M, Khan, M: Modified Laplace decomposition method. Appl. Math. Sci. (Ruse) 4(33-36), 1769-1783 (2010)

20. Khan, Y, Wu, Q: Homotopy perturbation transform method for nonlinear equations using He's polynomials. Comput. Math. Appl. 61(8), 1963-1967 (2011)

21. Kowalczyk, R, Forys, U: Qualitative analysis on the initial value problem to the logistic equation with delay. Math. Comput. Model. 35(1-2), 1-13 (2002) 